\title{
LA COMPRENSIÓN CONCEPTUAL ARITMÉTICA EN LA ESCUELA ELEMENTAL
}

\author{
Leire Pérez Pérez \\ Universidad de Vigo leiperez@uvigo.es \\ Andrea Núñez López \\ Universidad de Vigo nulopezandrea@gmail.com \\ Valentín Iglesias Sarmiento \\ Universidad de Vigo visarmiento@uvigo.es
}

Recepción Artículo: 27 octubre 2021

Admisión Evaluación: 27 octubre 2021

Informe Evaluador 1: 28 octubre 2021

Informe Evaluador 2: 29 octubre 2021

Aprobación Publicación: 30 octubre 2021

\section{RESUMEN}

Este estudio analizó la comprensión conceptual aritmética desde una doble vía, (conceptual y estratégica) del alumnado escolarizado en $4^{0}, 5^{\circ}$ y $6^{\circ}$ de Educación Primaria, seleccionado en base a tres grupos de logro: dificultades de aprendizaje en matemáticas (DAM; $n=51)$, competencia normal $(C N ; n=60)$ y alto rendimiento (AR; $\mathrm{n}=21$ ). Concretamente, pretendió (1) caracterizar al alumnado en base a su conocimiento conceptual aritmético y (2) analizar cómo los distintos grupos de logro afrontan tareas más complejas en base a sus habilidades conceptuales previas. Se utilizó la batería BANEVHAR para evaluar la comprensión conceptual y la escala completa de la batería CAS como estimador fiable de inteligencia. Los resultados señalaron los déficits conceptuales del alumnado con DAM respecto a sus iguales de CN y AR que parecen llegar a estos niveles educativos con las habilidades conceptuales adquiridas. Además, se observó que existen diferencias entre los tres grupos en el afrontamiento estratégico de las tareas cuando estas son novedosas o complejas. En este contexto, el alumnado con AR es capaz de resolver las tareas de forma más eficiente. Estos hallazgos resaltan la importancia de la enseñanza conceptual y estratégica de la aritmética y sugieren su implementación práctica en la escuela.

Palabras clave: comprensión conceptual aritmética; dificultades de aprendizaje en matemáticas (DAM); competencia normal (CN); alto rendimiento (AR); escuela elemental

\section{ABSTRACT}

Arithmetic conceptual understanding in elementary school. This study analyzed the arithmetic conceptual comprehension from a dual perspective (conceptual and strategic) of students in 4th, 5th and 6th grades of Elementary school, selected based on three achievement groups: mathematical learning disabilities (MLD; $n=51)$, typical achievement (TA; $n=60)$ and high achievement (HA; $n=21)$. Specifically, it aimed to (1) characterize 


\section{LA COMPRENSIÓN CONCEPTUAL ARITMÉTICA EN LA ESCUELA ELEMENTAL}

students based on their arithmetic conceptual knowledge and (2) analyze how the different achievement groups cope with more complex tasks based on their previous conceptual skills. The BANEVHAR battery was applied to assess conceptual understanding and the full scale of the CAS battery was used as a reliable estimator of intelligence. The results pointed out the conceptual deficits of students with MLD with respect to their peers with TA and $\mathrm{HA}$, who seem to reach these educational levels with acquired conceptual skills. In addition, it was observed that there are differences between the groups not only in conceptual knowledge but also in the strategic coping of tasks when these are novel or complex. In this context, students with HA can solve tasks more efficiently. These findings highlight the importance of conceptual and strategic teaching of arithmetic and suggest its practical implementation at school.

Keywords: arithmetic conceptual comprehension; mathematical learning disabilities (MLD); typical achievement (TA); high achievement (HA); elementary school

\section{INTRODUCCIÓN}

El estudio del rendimiento aritmético ha despertado gran interés a lo largo de los años, especialmente en edades tempranas y primeros cursos de la escuela elemental. Sin embargo, distintos informes internacionales de gran repercusión como el Trends in International Mathematics and Science Study (TIMMS; IEA, 2016) sitúan, en el contexto de la OCDE, en un nivel muy bajo al 7\% del alumnado de $4^{0}$ de educación primaria y en el otro extremo, en un nivel avanzado, al 3\%. Dada la relevancia del desarrollo aritmético dentro de la escuela elemental y su repercusión incluso para la empleabilidad en la vida adulta (Geary, 2011), parece importante entender los factores que subyacen al rendimiento aritmético diferencial de los niños en los últimos cursos de la escuela elemental.

La mayor parte de los estudios se han centrado en una amplia variedad de habilidades cognitivas (e.g. procesamiento simultáneo, memoria de trabajo) y habilidades de dominio específico (e.g. conteo, comprensión de la magnitud, place value). Un importante cuerpo de datos que ha recibido menor atención por parte de la literatura ha sido el referido a la implicación de la competencia conceptual en la aritmética. Este constructo de amplio espectro cuya definición no está suficientemente consensuada (Crooks y Alibali, 2014) se vincula en este trabajo a la comprensión de los principios y operaciones aritméticas y las leyes subyacentes de cada tipo de operación (Baroody, 2003). De forma operativa, la comprensión conceptual aritmética se establece como elemento clave en una segunda fase del desarrollo de la aritmética tras el conteo (Baroody, 2006) y como un elemento estratégico en el afrontamiento de tareas novedosas en las que los hechos son desconocidos y se ignoran los procedimientos de cálculo ordinarios, o bien éstos son demasiado complicados y hay presión temporal para resolver la tarea (Dowker, 2005). En este contexto se ha analizado a través del conocimiento de los símbolos aritméticos (Ploger y Hetch, 2009) y, de forma más concreta, acudiendo a la derivación

de respuestas aproximadas vinculadas al manejo de los principios aritméticos básicos 0 la descomposición de los números en partes (Baroody, 2006) y a las habilidades de estimación aritmética (Dowker, 2005).

La investigación en torno a la derivación de respuestas exactas apunta que el rendimiento aritmético con sumas y restas se relacionó en alumnos del $1^{0}$ y $2^{0}$ de la escuela elemental con su comprensión de los principios aritméticos y de las relaciones parte-todo (Canobi, 2004; Jordan et al., 2009). También que el rendimiento aritmético puede predecirse en $2^{\circ}$ y $3^{0}$ a partir de medidas vinculadas a los principios aritméticos, una vez controladas las habilidades cognitivas (Cowan et al., 2011). Nunes et al. (2007) proporcionan evidencia longitudinal de la capacidad predictora de una medida global de razonamiento lógico basada en los principios aritméticos, la composición aditiva y la seriación realizada al comienzo de $1^{0}$ respecto al rendimiento observado en una prueba curricular matemática 16 meses después, independientemente de rendimiento cognitivo previo. En todo caso, algunos estudios que analizan la relación entre la utilización de los principios aritméticos de inversión y el rendimiento en tareas de cálculo simple no han conseguido encontrar resultados satisfactorios (e.g., Sherman y Bisanz, 2007) lo que ha llevado a distintos autores a analizar la relación entre la comprensión de los principios y el rendimiento aritmético con sumas y restas a través del análisis de perfiles. En esta línea de investigación, se han encontrado combinaciones de perfiles entre el rendimiento aritmético y conceptual en edades que abarcan desde 
$1^{0}$ (Gilmore et al., 2017) hasta 300 de la escuela elemental (Gilmore y Bryant, 2006) que Gilmore y PapadatouPastou (2009) extienden en su metaanálisis hasta los 13 años.

Un segundo grupo de habilidades implicadas en la resolución de las tareas aritméticas se recogen tradicionalmente bajo la denominación de estimación aritmética. Para desplegar las respuestas aproximadas de forma eficiente, es necesario el dominio de varios tipos de comprensión conceptual (LeFevre et al., 1993; Siegler y Booth, 2005) y el conocimiento de hechos y procedimientos estratégicos (Dowker, 2005). Estas habilidades comienzan a desarrollarse tarde, de forma gradual (Siegler y Booth, 2005) y suelen mejorar entre el $3^{\text {er }}$ y el $6^{0}$ grado de la escuela elemental (LeFevre et al., 1993) con cambios hacia una mayor sistematización en las estrategias utilizadas en el último nivel (Lemaire y Lecacheur, 2002). En su estudio transversal con tareas de multiplicación con dígitos simples y multidígitos, LeFevre et al. (1993) se encontraron con que en $4^{0}$ solo el 20\% de los niños podían resolver con éxito las tareas de estimación propuestas, aunque ya en $6^{0}$ la mayor parte de los niños resolvían las tareas propuestas con éxito.

Sorprendentemente, se localizan pocas investigaciones referidas a la utilización de estrategias de hechos derivados en niños con dificultades aritméticas. Con todo, distintos estudios evolutivos han señalado que la mayor parte de los niños utilizan, desde edades tempranas, distintas estrategias de apoyo basadas en el conteo (Geary et al., 2004) y en hechos derivados (Dowker, 2005). Así, los niños emplean distintos principios conceptuales básicos como la propiedad conmutativa, la propiedad asociativa o los principios inversos suma/resta y multiplicación/división como soporte para resolver problemas aritméticos (Dowker, 2003). Estos hallazgos se han observado también en niños con desventajas educativas (Jordan Huttenlocher et al., 1994) y con dificultades aritméticas (Baroody y Snyder, 1983).

Por otro lado, en algunos estudios realizados en los últimos años, Jordan y colaboradores (e.g., Hanich et al., 2001 en 2; Jordan et al., 2003), han localizado a niños de corta edad con dificultades aritméticas que rendían a niveles inferiores sus iguales en tareas conceptuales que evalúan la estimación o los principios aritméticos. Esta relación entre los déficits conceptuales y las dificultades aritméticas apuntadas por la literatura (véase también Geary et al., 1992) no han podido ser confirmados hasta el momento en

niños de niveles educativos superiores (e.g., Mabbott y Bisanz, 2008). Dowker (2005) estudió las habilidades de estimación en niños de entre 6 y 7 años con dificultades aritméticas. Los resultados indicaron que los niños con dificultades realizaban las tareas de estimación aritméticas al mismo nivel que el grupo control. La autora señaló, al respecto, la no relación directa entre el mal rendimiento en cálculo y unas habilidades de estimación aritmética deficientes, ya que pueden encontrarse niños que alcanzan niveles normales en cálculo y muy por debajo de lo esperable en tareas de estimación aritmética. Macaruso y Sokol (1998) comunicaron hallazgos semejantes.

Respecto a la competencia conceptual del alumnado con alto rendimiento matemático los estudios son eminentemente descriptivos. Dowker (2005), en una investigación con niños de entre 5 y 9 años, encontraron que los niños con mayor éxito en tareas de aritmética mental con sumas mostraban una mayor utilización de las estrategias de hechos derivados respecto a sus iguales. En el caso de la estimación aritmética, Star et al. (2009) también vinculan el mayor rendimiento previo en alumnado de $5^{0}$ y $6^{\circ}$ con estrategias que conducían a estimaciones más precisas respecto al menor rendimiento asociado a estrategias menos sofisticadas.

\section{OBJETIVOS}

En este contexto, en este estudio se analizó la comprensión conceptual aritmética del alumnado escolarizado en los tres cursos finales de la Educación Primaria española $\left(4^{0}, 5^{\circ}\right.$ y $\left.6^{0}\right)$ organizados en base a su rendimiento aritmético previo. De forma concreta, se contempló la comprensión conceptual aritmética desde una doble vía: conceptual y estratégica. En esta línea, se trató de (1) caracterizar a alumnado con distintos niveles logro (DAM, CN y AR) en base a su conocimiento conceptual aritmético y (2) analizar cómo los distintos grupos de logro afrontan tareas más complejas en base a sus habilidades conceptuales previas. 


\section{LA COMPRENSIÓN CONCEPTUAL ARITMÉTICA EN LA ESCUELA ELEMENTAL}

\section{MUESTRA}

La muestra abarcó un total de 132 alumnos escolarizados en $4^{0}(n=48)$, 5ำ $(n=43)$ y $6^{0}(n=41)$ de Educación Primaria en ocho centros pertenecientes a la comunidad gallega. La muestra incluía 73 niños (55,30\%) y 59 niñas $(44,70 \%)$, sin evidencias de necesidades educativas especiales y con edades comprendidas entre los 9 y 12 años.

El criterio operacional para la selección de los 51 sujetos (20 de cuarto, 17 y 14 de sexto) del grupo con DAM fue una puntuación estándar igual o menor que 85 en la escala de cálculo de la BANEVHAR (Iglesias-Sarmiento, 2009). Para la selección de los 60 niños (20 de cada nivel educativo) con CN se estableció como criterio cuantitativo la obtención de puntuaciones estándar entre 86 y 114 en la escala de cálculo de la BANEVHAR. Finalmente, se asignaron 21 niños (8 de cuarto, 6 de quinto y 7 se sexto) al grupo AR a partir de una puntuación estándar igual o superior a 115 en la escala de cálculo de la BANEVHAR.

\section{METODOLOGÍA}

\section{Medidas}

\section{Tareas de selección}

Para la asignación de los sujetos a los distintos grupos de rendimiento se utilizó la puntuación obtenida en la escala de cálculo de la batería BANEVHAR (Iglesias- Sarmiento, 2009). Esta escala tiene una fiabilidad de .84.

\section{Tarea de control}

Se aplicó la adaptación española de la batería CAS (Deaño, 2005) tomando como estimador fiable de la inteligencia la puntuación normalizada obtenida en la Escala Completa. Los coeficientes de fiabilidad oscilan desde .93 a .97 .

\section{Tareas experimentales}

Se administraron cuatro tareas de la escala dedicada a la Compresión Conceptual Aritmética de la BANEVHAR (Iglesias-Sarmiento, 2009). Las tres primeras tareas están relacionadas con el conocimiento conceptual: Verificación de operaciones, comprensión de los principios aritméticos básicos y cálculo aproximado, que evalúan el conocimiento de los símbolos, los principios básicos y la estimación aritmética, respectivamente. La cuarta tarea, rutinas matemáticas, está relacionada con el conocimiento estratégico y evalúa la capacidad de los niños de adaptarse a tareas complejas y/o novedosas utilizando su capacidad conceptual de base. La fiabilidad de esta escala fue de .75 .

\section{Procedimiento}

Todos los niños fueron evaluados en su colegio de origen, tras el consentimiento de padres y centro educativo. Se llevó a cabo de forma individual y en dos sesiones distintas para cada niño. En la primera sesión se administraron las tareas de la BANEVHAR y en la segunda se pasó la batería CAS, siguiendo de forma específica las instrucciones recogidas en las mismas. El promedio de duración se situó en torno a 2 horas por alumno.

\section{Análisis de datos}

En la Tabla 1 se presentan las puntuaciones de los niños organizadas por grupo de rendimiento aritmético y por nivel educativo en cada una de las variables conceptuales. Debido a que la muestra incluye a niños de diferentes grupos de edad y con distintos niveles de medida, las puntuaciones directas se transformaron a puntuaciones z, estandarizadas por nivel educativo. Estas puntuaciones se emplearon en los distintos análisis realizados.

Los resultados fueron analizados a partir de distintos análisis de covarianza (ANCOVA) en los que se manejaron como variables independientes el grupo de competencia aritmética (DAM, CN, AR) y el nivel educativo (4ํㅜㄴ, 
$5^{0}$ y $\left.6^{0}\right)$. Como variables dependientes se utilizaron las puntuaciones alcanzadas en las tareas de comprensión conceptual (verificación de operaciones, comprensión de los principios aritméticos básicos, cálculo aproximado y rutinas matemáticas). Se añadió como covariables el nivel de funcionamiento cognitivo y la edad de los participantes, con el fin de controlar su efecto. Para los análisis post hoc se recurrió al método Bonferroni.

El análisis estadístico de las variables se llevó a cabo con la aplicación informática Statistical Product and Service Solutions (SPSS) en su versión 21.

\section{RESULTADOS}

Los resultados obtenidos en la escala completa de comprensión conceptual, mostraron efectos significativos del grupo de logro, $F(2,121)=48.22, p<.001,2$ parcial $=.444$ y del funcionamiento cognitivo, $F(1,121)=12.99$, $p<.001,{ }^{2}$ parcial $=.097$

Los análisis post hoc mostraron que el grupo con DAM rindió significativamente por debajo del grupo con CN $(M=-13.73, p<.001)$ y AR $(M=-30.25, p<.001)$. Además, se encontraron diferencias significativas entre el grupo de CN y el grupo con AR $(M=-16.51, p<.001)$.

\section{Conocimiento de símbolos}

Los resultados mostraron efectos significativos del grupo de logro, $F(2,121)$

$=14,59, p<.001,{ }^{2}$ parcial $=.194$ y del funcionamiento cognitivo, $F(1,121)=4.47, p<.05,2$

parcial $=.036$.

Los contrastes post hoc mostraron que el grupo con DAM obtuvo puntuaciones significativamente más bajas que el grupo con $\mathrm{CN}(M=-82, p<.001)$ y $\mathrm{AR}(M=-1.12, p<.001)$.

\section{Principios aritméticos}

Los resultados mostraron efectos significativos del grupo de logro, $F(2,121)=17.82, p<.001,{ }^{2}$ parcial $=.228$ y del funcionamiento cognitivo, $F(1,121)=5.55, p<.05,{ }^{2}$ parcial $=.044$.

Los resultados de los análisis post hoc mostraron que el grupo de DAM obtuvo puntuaciones significativamente más bajas que el grupo con $\mathrm{CN}(M=-.88, p<.001)$ y $\mathrm{AR}(M=-1.18, p<.001)$.

\section{Estimación}

Los resultados mostraron efectos significativos del grupo de logro, $F(2,121)=11.42, p<.001,{ }^{2}$ parcial $=.159$ y del funcionamiento cognitivo, $F(1,121)=4.70, p<.05,2$

parcial= $=037$.

La comparación entre los grupos de logro reveló que el grupo con DAM rindió significativamente por debajo del grupo con CN ( $M=-.71, p<.001)$ y $\mathrm{AR}(M=-1.07, p<.001)$.

\section{Tareas complejas}

El análisis reveló efectos significativos del grupo de logro, $F(2,121)=8.01, p=.001,{ }^{2}$ parcial=.117 y del grado educativo, $F(2,121)=33.00, p<.001,{ }^{2}$ parcial $=.353$.

Los análisis post hoc mostraron un rendimiento significativamente más alto del grupo de AR respecto a los grupos con DAM ( $M=2.00, p<.001)$ y con $\mathrm{CN}(M=1.65, p<.01)$. No se encontraron diferencias significativas entre el rendimiento de los niños con DA y CN $(p<.05)$. Además, los resultados mostraron que los alumnos de $6^{0}$ obtuvieron un rendimiento significativamente más alto que los de $4^{0}(M=3.09, p<.001)$ y $5^{\circ}(M=2.87, p<$ $.001)$. 


\section{LA COMPRENSIÓN CONCEPTUAL ARITMÉTICA EN LA ESCUELA ELEMENTAL}

Tabla 1

Estadísticos descriptivos para las habilidades conceptuales por tareas y grupos de rendimiento aritmético

\begin{tabular}{|c|c|c|c|c|c|}
\hline \multirow[b]{3}{*}{ Comp. conceptual } & \multirow[t]{3}{*}{ Curso } & \multicolumn{3}{|c|}{ Grupo } & \multirow[b]{2}{*}{ Total Curso } \\
\hline & & $\begin{array}{l}\text { DAM } \\
n=51\end{array}$ & $\begin{array}{c}\mathrm{CN} \\
\mathrm{n}=60\end{array}$ & $\begin{array}{c}\text { AR } \\
n=21\end{array}$ & \\
\hline & & $M(S D)$ & $M(S D)$ & $M(S D)$ & $M(S D)$ \\
\hline \multirow[t]{4}{*}{ Símbolos. } & $4^{\circ}$ & $-.54(1.01)$ & $.12(.88)$ & $.91(.25)$ & $-.02(1.00)$ \\
\hline & $5^{\circ}$ & $-.81(1.15)$ & $.43(.53)$ & $.53(.30)$ & $-.05(1.02)$ \\
\hline & $6^{\circ}$ & $-.73(1.26)$ & $.25(.72)$ & $.58(.00)$ & $-.03(1.02)$ \\
\hline & $\begin{array}{l}\text { Total } \\
\text { Grupo }\end{array}$ & $-.68(1.11)$ & $.27(.72)$ & $.69(.27)$ & $-.03(1.00)$ \\
\hline \multirow[t]{4}{*}{ Principios } & $4^{\circ}$ & $-.85(.56)$ & $.36(.80)$ & $1.07(.53)$ & $-.03(.99)$ \\
\hline & $5^{\circ}$ & $-.60(1.08)$ & $.20(.89)$ & $.65(.27)$ & $-.05(1.01)$ \\
\hline & $6^{\circ}$ & $-.68(1.31)$ & $.27(.69)$ & $.43(.39)$ & $-.03(1.02)$ \\
\hline & $\begin{array}{l}\text { Total } \\
\text { Grupo }\end{array}$ & $-.72(.97)$ & $.28(.78)$ & $.74(.49)$ & $-.04(1.00)$ \\
\hline \multirow[t]{4}{*}{ Estimación } & $4^{\circ}$ & $-.57(.88)$ & $.09(.86)$ & $1.07(.63)$ & $-.02(1.00)$ \\
\hline & $5^{\circ}$ & $-.68(1.07)$ & $.34(.71)$ & $.55(.75)$ & $-.04(1.01)$ \\
\hline & $6^{\circ}$ & $-.56(1.22)$ & $.20(.86)$ & $.48(.44)$ & $-.01(1.02)$ \\
\hline & $\begin{array}{l}\text { Total } \\
\text { Grupo }\end{array}$ & $-.61(1.03)$ & $.21(.80)$ & $.73(.64)$ & $-.02(1.00)$ \\
\hline \multirow[t]{4}{*}{ Tareas complejas } & $4^{\circ}$ & $.35(.99)$ & $.55(1.23)$ & $2.50(2.83)$ & $.79(1.68)$ \\
\hline & $5^{\circ}$ & $.53(.80)$ & $1.10(1.97)$ & $2.33(2.07)$ & $1.05(1.69)$ \\
\hline & $6^{\circ}$ & $3.14(2.03)$ & $3.60(2.46)$ & $6.00(1.29)$ & $3.85(2.34)$ \\
\hline & $\begin{array}{l}\text { Total } \\
\text { Grupo }\end{array}$ & $1.18(1.77)$ & $1.75(2.34)$ & $3.62(2.71)$ & $1.83(2.34)$ \\
\hline
\end{tabular}

\section{DISCUSIÓN}

En este estudio focalizado en los tres niveles finales de la Educación Primaria se trató de caracterizar, en base a su conocimiento conceptual, a alumnado con distintos niveles de logro aritmético (DAM, CN y AR) y de analizar cómo los distintos grupos en base a las estrategias adquiridas afrontan tareas novedosas o complejas.

Respecto al primer objetivo, vinculado al conocimiento conceptual, los datos revelan diferencias significativas entre los grupos de logro. Como era de esperar, los niños con DAM rinden significativamente por debajo de Ios niños con CN y AR. De forma concreta muestran un déficit conceptual respecto a sus iguales con CN y AR tanto en el conocimiento de los símbolos y principios aritméticos como en la derivación de respuestas aproximadas. Estos datos extienden a estos niveles educativos los resultados de Jordan y colaboradores (e.g., Hanich et al., 2001 en 2:; Jordan et al., 2003) que localizaron en los primeros niveles educativos, un rendimiento inferior a sus iguales en tareas que evalúan la estimación o los principios aritméticos.

Por otro lado, en contraste con lo establecido por Lefevre et al. (1993), Ios ANCOVAS no denotan una progresión en la adquisición de estos conceptos en estos niveles educativos. Una posible interpretación de estos 
resultados es que los niños con DAM llegan a $4^{0}$ curso de la Educación Primaria con déficits conceptuales que se mantienen hasta finales de la Educación Primaria mientras que sus compañeros con CN y AR ya dominan estos conocimientos en el $4^{\circ}$ curso.

Un dato interesante de este este estudio es que no se localizaron diferencias significativas en el conocimiento conceptual de la aritmética entre los grupos con CN y AR. En este sentido, debe de incidirse que en nuestro estudio se controló el funcionamiento cognitivo a través de una prueba global como el CAS que abarca medidas ejecutivas y de procesamiento, por lo que parece interesante analizar este efecto en futuras investigaciones, especialmente si se utilizan tareas matemáticas de carácter novedoso 0 más complejas (véase Iglesias-Sarmiento et al. 2020 para discusión).

El hallazgo principal de este estudio se vincula al segundo objetivo, cuando se analiza cómo los distintos grupos afrontan tareas no rutinarias y novedosas en las que los hechos son desconocidos y se ignoran los procedimientos de cálculo ordinarios (Dowker, 2005). En este contexto, afloran las diferencias entre los tres grupos de logro. Como anteriormente, el grupo con DAM muestra un menor rendimiento que puede vincularse tanto a sus dificultades aritméticas como a su bajo nivel conceptual. Por su parte, el grupo con AR es el que tiene más éxito a la hora de afrontar este tipo de tareas. Lo interesante aquí es su comparación con el grupo de CN, ya que ambos mostraban un conocimiento conceptual semejante en lo vinculado a los principios y a la derivación de respuestas aproximadas. Sin embargo, cuando la tarea implica la aplicación estratégica el grupo con AR muestra una mejor utilización de las estrategias conceptuales a la hora de afrontar la tarea. Estos datos extienden los escasos estudios que vinculaban al alumnado con AR con el uso de un espectro más amplio de estrategias de hechos derivados respecto a sus iguales (Dowker, 2005) y el uso de estrategias más sofisticadas (Star et al., 2009).

\section{CONCLUSIONES}

Los resultados de este estudio aportan algunos datos de interés educativo. Por un lado, parece que el alumnado con DAM no domina distintos aspectos conceptuales esenciales de la aritmética en los niveles finales de la Educación Primaria respecto a sus iguales de CN y AR que parecen llegar a estos niveles educativos con el conocimiento conceptual ya adquirido. Por otro lado, se observó, una vez controlado el funcionamiento cognitivo, que existen diferencias entre los grupos no solo en el conocimiento conceptual sino en el afrontamiento estratégico de las tareas cuando estas son novedosas o complejas. En este contexto, el alumnado con AR es capaz de resolver las tareas de forma más eficiente. La caracterización de un grupo con AR no superdotado debe de tenerse en

cuenta para futuras investigaciones y en lo que respecta a la enseñanza de conceptos y estrategias y a su implementación práctica en la escuela.

En todo caso, los resultados deben considerarse en base a sus propias limitaciones. La primera deviene del tamaño de la muestra de los grupos. Otra limitación del estudio tiene que ver con el tratamiento transversal de los datos. Del mismo modo, los resultados deben interpretarse al amparo de los instrumentos utilizados que pueden provocar diferencias entre los estudios.

\section{REFERENCIAS BIBLIOGRÁFICAS}

Baroody, A. J. (2003). The development of adaptive expertise and flexibility: The integration of conceptual and procedural knowledge. En A. J. Baroody y A. Dowker (Ed.), The development of arithmetic concepts and skills: Constructing adaptive expertise (pp. 1-33). Erlbaum.

Baroody, A. J. (2006). Why children have difficulty mastering the basic number combinations and how to help them. Teaching Children Mathematics, 13(1), $22-31$.

Baroody, A. J., y Snyder, P. (1983). A cognitive analysis of basic arithmetic abilities of TMR children. Education and Training of the Mentally Retarded, 18(4), 253-259. Canobi, K. H. (2004). Individual differences in children's addition and subtraction knowledge. Cognitive Development, 19(1), 81-93.

https://doi.org/10.1016/j.cogdev.2003.10.001 


\section{LA COMPRENSIÓN CONCEPTUAL ARITMÉTICA EN LA ESCUELA ELEMENTAL}

Cowan, R., Donlan, C., Shepherd, D.-L., Cole-Fletcher, R., Saxton, M., y Hurry, J. (2011). Basic calculation proficiency and mathematics achievement in elementary school children. Journal of Educational Psychology, 103(4), 786-803. http://dx.doi.org/10.1037/a0024556

Crooks, N. M., y Alibali, M. W. (2014). Defining and measuring conceptual knowledge in mathematics. Developmental Review, 34 (4), 344-377. http://dx.doi.org/10.1016/j.dr.2014-10-001

Deaño, M. (2005). D.N: CAS (Das-Naglieri: Sistema de Evaluación Cognitiva) Adaptación Española. Gersam.

Dowker, A. (2003). Young children's estimates for addition: The zone of partial knowledge and understanding. En A. J. Baroody (Ed.), The development of arithmetic concepts and skills: Constructing adaptive expertise (pp. 243-265). Erlbaum. Dowker, A. D. (2005). Individual differences in arithmetic. Implications for Psychology, Neuroscience and Education. Psychology Press.

Geary, D. C. (2011). Cognitive predictors of individual differences in achievement growth in mathematics: A five year longitudinal study. Developmental Psychology, 47(6), 1539-1552. http://dx.doi.org/10.1037/a0025510

Geary, D. C., Bow-Thomas, C. C., y Yao, Y. (1992). Counting knowledge and skill in cognitive addition: A comparison of normal and mathematically disabled children. Journal of Experimental Child Psychology, 54(3), 372-391. https://doi.org/10.1016/0022-0965(92)90026-3

Geary, D. C., Hoard, M. K., Byrd-Craven, J., y Catherine DeSoto, M. (2004). Strategy choices in simple and complex addition: Contributions of working memory and counting knowledge for children with mathematical disability. Journal of Experimental Child Psychology, 88(2), 121-151. https://doi.org/10.1016/j.jecp.2004.03.002

Gilmore, C. K., y Bryant, P. (2006). Individual differences in children's understanding of inversion and arithmetical skill. The British Journal of Educational Psychology, 76(2), 309-331. https://doi.org/10.1348/000709905X39125

Gilmore, C., Keeble, S., Richardon, S., y Cragg, L. (2017). The interaction of procedural skill, conceptual understanding and working memory in early mathematics achievement. Journal of Numerical Cognition, 3(2), 400416. https://doi.org/10.5964/jnc.v3i2.51

Gilmore, C. K., y Papadatou-Pastou, M. (2009). Patterns of individual differences in conceptual understanding and arithmetical skill: A meta-analysis. Mathematical Thinking and Learning, 11(1-2), 25-40. http://dx.doi.org/10.1080/10986060802583923

Hanich, L. B., Jordan, N. C., Kaplan, D., y Dick, J. (2001). Performance across different areas of mathematical cognition in children with learning difficulties. Journal of Educational Psychology, 93(3), 615-626. https://doi.org/10.1037/0022-0663.93.3.615

Iglesias-Sarmiento, V. (2009). Dificultades de aprendizaje en el dominio aritmético y en el procesamiento cognitivo subyacente [Learning difficulties in the mastery of arithmetic and in the underlying cognitive processing]. (Doctoral dissertation). Available from ProQuest database. (UMI No. AAT 3386296)

Iglesias-Sarmiento, V., Alfonso, S., Conde, A., Pérez, L., y Deaño, M. (2020). Mathematical difficulties vs. high achievement: An analysis of arithmetical cognition in elementary school.

Developmental Neuropsychology, 45(2), 49-65.

https://doi.org/10.1080/87565641.2020.1726920

International Association for the Evaluation of Educational Achievement (IEA, 2016). TIMMS 2015. Student achievement. Chestnut Hill, MA: TIMSS \& PIRLS International Study Center.

Jordan, N. C., Hanich, L. B., y Kaplan, D. (2003). Arithmetic fact mastery in young children: A longitudinal investigation. Journal of Experimental Child Psychology, 85(2), 103-119. https://doi.org/10.1016/S00220965(03)00032-8

Jordan, N. C., Huttenlocher, J., y Levine, S. C. (1994). Assessing early arithmetic abilities: Effects of verbal and nonverbal response types on the calculation performance of middle-and low-income children. Learning and Individual Differences, 6(4), 413-432. https://doi.org/10.1016/1041-6080(94)90003-5 
Jordan, J.-A., Mulhern, G., y Wylie, J. (2009). Individual differences in trajectories of arithmetical development in typically achieving 5- to 7-year-olds. Journal of Experimental Child Psychology, 103(4), 455-468. https://doi.org/10.1016/j.jecp.2009.01.011

LeFevre, J.- A., Greenham, S. L., y Waheed, N. (1993). The development of procedural and conceptual knowledge in computational estimation. Cognition and Instruction, 11(2), 95-132. http://dx.doi.org/10.1207/s1532690xci1102_1

Lemaire, P., y Lecacheur, M. (2002). Children's strategies in computational estimation. Journal of Experimental Child Psychology, 82(4), 281-304. https://doi.org/10.1016/S0022-0965(02)00107-8

Mabbott, D. J., y Bisanz, J. (2008). Computational skills, working memory, and conceptual knowledge in older children with mathematics learning disabilities. Journal of Learning Disabilities, 41(1), 15-28. https://doi.org/10.1177/0022219407311003

Macaruso, P., y Sokol S. M. (1998). Cognitive neuropsychology and developmental dyscalculia. En C. Donlan (Ed.), The development of mathematical skills (pp. 201- 205). Psychology Press.

Nunes, T., Bryant, P., Evans, D., Bell, D., Gardner, S., Gardner, A., y Carraher, J. (2007). The contribution of logical reasoning to the learning of mathematics in primary school. British Journal of Developmental Psychology, 25(1), 147- 166. https://doi.org/10.1348/026151006X153127

Ploger, D., y Hecht, S. (2009). Enhancing children's conceptual understanding of mathematics through Chartworld software. Journal of Research in Childhood Education, 23(3), 267-277. https://doi.org/10.1080/02568540909594660

Sherman, J., y Bisanz, J. (2007). Evidence for use of mathematical inversion by three- year-old children. Journal of Cognition and Development, 8(3), 333-344. https://doi.org/10.1080/15248370701446798

Siegler, R. S., y Booth, J. L. (2005). Development of numerical estimation: A review. En

J. I. D. Campbell (Ed.), Handbook of mathematical cognition (pp. 197-212). Psychology Press.

Star, J. R., Rittle-Johnson, B., Lynch, K., y Perova, N. (2009). The role of prior knowledge in the development of strategy flexibility: the case of computational estimation. Mathematics Education, 41 (5), 569-579.

http://dx.doi.org/10.1007/s11858-009-0181-9 
